\title{
Beam Transfer Lines for the Spallation Neutron Source*
}

\author{
D. Raparia, Y. Y. Lee, W. T. Weng and J. Wei \\ Brookhaven National Laboratory \\ P. O. Box 5000, Upton, NY 11793, Upton, USA
}

\begin{abstract}
Beam transfer lines for the Spallation Neutron Source (SNS) are designed to have low beam losses for hand on maintenance while satisfying the facility footprint requirements. There are two main beam transfer lines, High Energy Beam Transport (HEBT) line which connect super conducting linac to the accumulator ring and Ring to Target Beam transport (RTBT) which transfers beam from accumulator ring to the target. HEBT line not only transfer the beam from linac to ring but also prepare beam for ring injection, correct the energy jitter from the linac, provide required energy spread for the ring injection, clean the transverse and longitudinal halo particles from the beam, determine the linac beam quality, and provide the protection to the accumulator ring. RTBT line transport the beam from ring to target while fulfilling the target requirements of beam size, maximum current density, beam moment on the target in case of ring extraction kicker failure, and protect the target from the ring fault conditions.
\end{abstract}

\section{INTRODUCTION}

The 1.4 MW Spallation Neutron Source (SNS) is under construction in Oak Ridge National Laboratory, order of magnitude higher power than any other existing machines. The whole facility is design for low un-controlled beam losses for hand on maintenance. The SNS[1] consists $1.0 \mathrm{GeV}$ super conducting Linac[2] and an accumulator ring[3] and two main transfer lines. High Energy Beam Transfer (HEBT) line [4] connects super conducting Linac to the accumulator ring and Ring to Target Beam Transfer (RTBT) line[5] connects accumulator ting to the target. These lines are carefully designed to have low uncontrolled beam losses with several new features in these lines like energy corrector and spreader cavities, collimators etc.

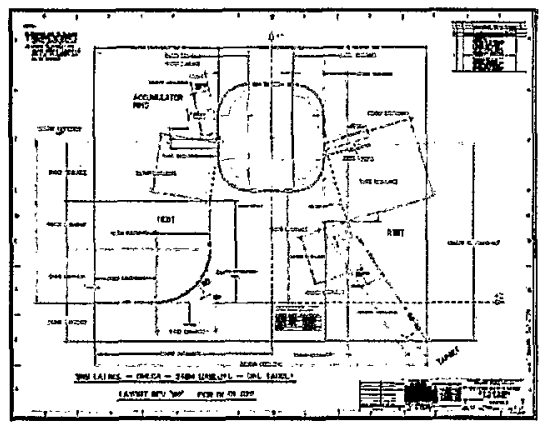

FIGURE 1. SNS transfer lines and ring layout.

\section{HIGH ENERGY BEAM TRANSFER LINE}

HEBT is about 180 meter long and carry $\mathrm{H}$ ion with peak average current of $38 \mathrm{~mA}$ in $1 \mathrm{~ms}$ long pulses at the rate of $60 \mathrm{~Hz}$. The HEBT not only carry the $\mathrm{H}^{-}$ion *Work performned under the auspices of the U.S. Department of Energy. CP642, High Intensity and High Brightness Hadron Beams: $20^{\text {th }}$ ICFA Advanced Beam Dynamics Workshop on High Intensity and High Brightness Hadron Beams, edited by W. Chou, Y. Mori, D. Neuffer, and J.-F. Ostiguy (C) 2002 American Institute of Physics 0-7354-0097-0/02/\$19.00 but also optically matches linac and ring, correct the energy jitter from the linac, increase the energy spread of beam to avoid beam stability in the ring, clean the transverse and longitudinal halo coming from the linac, characterize the beam from linac, and protect ring from the fault conditions. The general features of this line are, the magnetic filed in dipole and quadrupoles and vacuum are low enough to control Lorentz and gas stripping of $\mathrm{H}$ for energies $800-1300$ $\mathrm{MeV}[6]$. The ratio of aperture to the rms beam size is kept greater than 10 through out the line except energy corrector, spreader and collimators. Figure 1 shows the layout of the HEBT, Ring and RTBT. For ease of operation and stability reasons the lattice of the is chosen is FODO which matches the linac and ring doublet-FODO lattices. Figure 2 shows the amplitude function $\left(\beta_{x}, \beta_{y}\right)$ and the dispersion function $(\eta)$ along the HEBT. We can consider the HEBT as having three sections: Linac-Achromat Matching Section (LAMS), Achromat, and Achromat-Ring Matching Section (ARMS). In addition to the bend to the ring, there is a straight beam line used for linac beam characterization, as shown in Fig. 1. The first five cells $(8 \mathrm{~m} / \mathrm{cell})$ after the linac (LAMS) are used to characterize the linac beam, match beam into the achromat, collimate beam halo. The energy corrector cavity is located in the last half cell of the LAMS. Following this, the four cell long achromat $(14 \mathrm{~m} / \mathrm{cell})$ provides momentum selection by cleaning up the beam energy halo at the point of maximum dispersion $\left(\eta_{x}=6.4 \mathrm{~m}\right)$. The remaining six cells $(8 \mathrm{~m} / \mathrm{cell})$ are used for matching the beam into the accumulator ring, diagnostics. The energy spreader cavity is located in the first cell following the achromat (in the ARMS), where the dispersion and its derivative are zero. There are eight horizontal and eight vertical dipole correctors placed in strategic positions of small apertures in the 
line. The line has following new features to control losses.

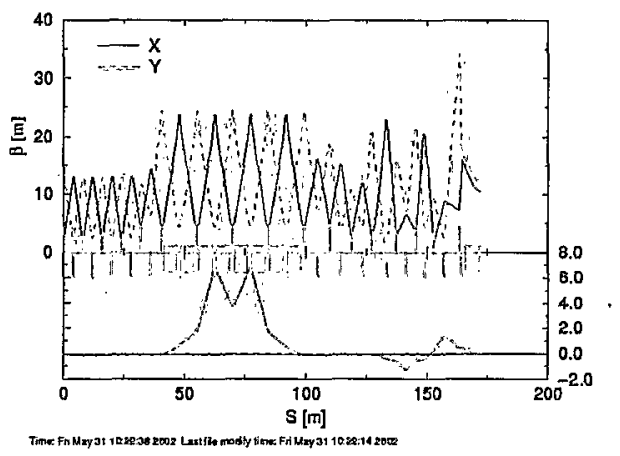

FIGURE 2. The $\beta_{\mathrm{x}}, \beta_{\mathrm{y}}$ and $\eta_{\mathrm{x}}$ along the HEBT.

\section{Transverse and Longitudinal Collimators}

To remove the transverse and longitudinal halo from the beam, we introduce adjustable carbon foils in the path of beam to intercept the tails. After the passage the $\mathrm{H}^{-}$ion through the foil, the two electrons are removed and the $\mathrm{H}^{-}$focusing lattice deviates the proton tails into large massive absorbers. There are a total of five set of such adjustable foil. Four transverse ( 2 each in $x$ and $y$ ) are located just after linac and fifth one is located in the achromat at the high dispersion[7]

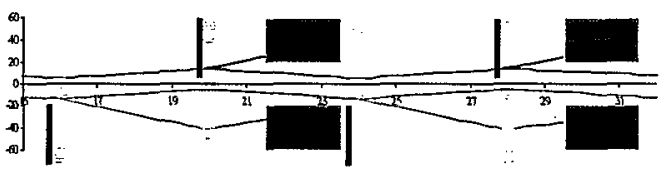

FIGURE 3. Carbon foils and absorber layout in HEBT.

Figure 3 shows the layout of the adjustable foils and absorbers. The phase advance between adjustable foils is $90^{\circ}$ (in each plane) providing the most efficient cut of the phase space. For $\mathrm{H}^{-}$collimation by charge exchange the interception of the secondary halo by the absorber is $100 \%$ efficient. Still, due to small impact parameters, the protons have a finite probability of escaping the absorber without being removed. We simulate the passage of protons through the material of the absorber with Monte Carlo code. The absorption efficiency for the secondary protons ranges 88 to 95 $\%$.

For longitudinal halo collimation, a charge exchange foil is located in high dispersion region in the HEBT achromat to remove the longitudinal tails from the beam before entering the ring. After being stripped, the protons are deviated by the dipole filed into a large absorber outside the beam trajectory. The vacuum pipe geometry after foil has been adjusted to clear the dipole and drive the protons on the absorber. In this case, the absorption efficiency in the collimator is $100 \%$, as protons are deviated from the main path and hit the absorber located outside the accelerator.

\section{Energy Corrector and Spreader Cavity}

Beam from the linac will have $2.0 \mathrm{MeV}$ energy jitter and $3.5^{\circ}$ for phase jitter due to $0.5^{\circ}$ and $0.5 \%$ amplitude control error in linac. To remove these phase and amplitude jitter an $805 \mathrm{MHz}$ (linac frequency) RF cavity is place just before the achromat which is phase lock to the linac. The particle due to energy difference will suffer the phase slip with respect to design momentum given by

$$
\Delta \phi_{L} \equiv \frac{\gamma}{\gamma(\gamma+1)} \frac{\Delta T}{T} \frac{L}{\beta c} 2 \pi f
$$

where $\beta, \gamma$ are the relativistic parameters, $T$ is the design kinetic energy and $f$ is the frequency and $l$ is length for the phase slip. The required voltage is given by $V_{0}=\Delta E / \sin \left(\phi_{\text {slip }}\right)$. The energy jitter after the corrector cavity $0.2 \mathrm{MeV}$ was achieved which is within the requirement of the ring.

SNS accumulator ring also need $\pm 4 \mathrm{MeV}$ energy spread for stability reason. This is achieved by placing another RF cavity after the achromat which frequency is about $100 \mathrm{kHz}$ different than the linac frequency. The linac bunches see different phase as they arrive at this cavity and gain/loss different energies depending on the cavity voltage, hence creating required energy spread without creating energy tails.

\section{RING TO TARGET TRANSFER LINE}

Ring to Target Beam Transfer (RTBT) line is about 150 meter long and carry the beam from the ring extraction region to the target and provide the desired footprint for the accelerator complex. The general features of this line are, the line is immune to one kicker failure and the ratio of acceptance to $\mathrm{rms}$ emittance is more than 20. Figure 4 shows the amplitude and the dispersion function along the RTBT.

The line has following function (a) extraction (b) beam dump (c) protect from device failure (d) beam spreader and (e) diagnostics. Following the extraction system beam can be dumped straight through a $16.8^{\circ}$ dipole magnet. After the magnet two cell are used for the collimator system which protect line from the dipole failure. Following another six cells of transport, the last five quadrupoles in the line are used for final beam spreading to produced the beam sized required at the target. 
1SNS RING TO TARGET TRANSFER LINE (03/01

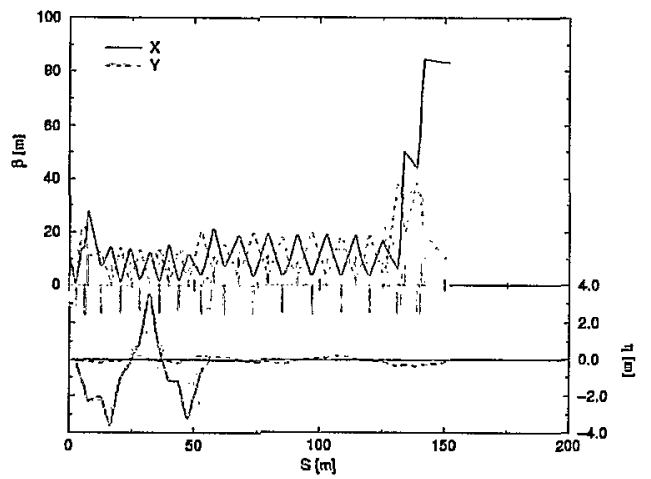

FIGURE 4. The $\beta_{x}, \beta_{y}$ and $\eta_{x}, \eta_{y}$ along the RTBT.

The phase advance between kickers and target is $\mathrm{n} \pi$, so that in event of a kicker failure beam will not move at the target. Figure 5 shows the closed orbit for different kickers failure.

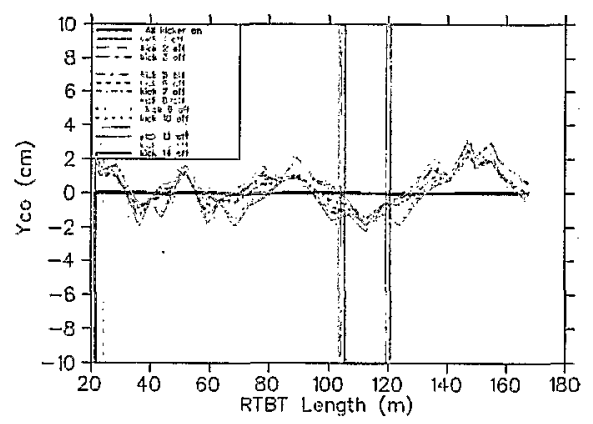

FIGURE 5. Close orbit due to kickers failure.

\section{Extraction}

The extraction of the beam is done in a single turn with full aperture at a pulse repetition frequency of 60 $\mathrm{Hz}$. The extraction system consists of two sets (seven each) kicker magnets and a Lambertson magnet septum and a dipole magnet. The Lambertson septum magnet will receive the vertically kicked down beam and will provide large deflection angle $\left(16.8^{\circ}\right)$ to enable ejection horizontally from the accumulator ring. A dipole, which is $540^{\circ}$ phase advance away from the Lamberton magnet, bends the beam horizontally in the same direction by $16.8^{\circ}$, making the extraction system achromatic. The Lambertson magnet is rotated $2.55^{\circ}$ anti-clock wise to neutralize the vertical kick from the kickers and making the beam about 9 inches difference between the ring and RTBT beam height.

\section{Beam Spreader}

The beam spreader consists of five radiation hard quadrupoles near the end of the RTBT. These five 36 $\mathrm{cm}$ diameter aperture quadrupoles provide the desired beam size at the target. Due to thermal considerations of the target, the beam current density on the target must remain below $0.25 \mathrm{~A} / \mathrm{m}^{2}$. This requirement results in a non-Gaussian beam distribution in space (with un-normalized rms emittance of $24 \pi \mathrm{mm}$ mrad). The required current density distribution is achieved using painting scheme described elsewhere[8].

\section{Beam collimation}

The RTBT acceptance is $480 \pi \mathrm{mm} \mathrm{mrad}$ and the expected beam emittance (100\%) from the ring is 240 $\pi \mathrm{mm}$ mrad. The line is design such a way that if one of fourteen kicker fail beam will pass through the line without scraping. To protect the line from device failures such as more than one kicker or dipole failure, there are two collimators $90^{\circ}$ apart in phase advance are placed just after the $16.8^{\circ}$ dipole. The acceptances of these collimators are 300 and $400 \pi \mathrm{mm} \mathrm{mrad}$ in horizontal and vertical plane respectively.

\section{REFERENCES}

1. N. Holkamp, "The SNS Linac and Storage Ring: Challenges and Progress Towards Meeting Them", EPAC 2002.

2. S. Nath, et al., "Selected Aspect of SNS Linac", EPAC 2002

3. J. Wei, et al., "Low-Loss Design for the High Intensity Accumulator Ring of the Spallation Neutron Source", PRST-AB, 3, 080101, pp. 1-19,2001

4. D. Raparia, et al., "The NSNS High Energy Beam Transport Line", Proc. 1997 Particle Accelerator Conference, p. 162.

5. D. Raparia, et al., "The SNS Ring to Target Beam Transport Line", Proc. 1999 Particle Accelerator Conference, p. 1297.

6. D. Raparia, et al., "Dependence of the SNS Transfer lines and Accumulator Ring on the Linac Energy", Proc. 2001 Particle Accelerator Conference, p. 3260.

7. N. Catalan-Lasheras, and D. Raparia, "The Collimation System of the SNS Transfer Lines, Proc. 2001 Particle Accelerator Conference, p. 3263.

8. J. Beebe-Wang, et al., "Transverse Phase Space Painting for SNS Accumulator Ring Injection", , Proc. 1999 Particle Accelerator Conference, p. 1743. 